\title{
ON THE EIGENVALUES OF POSITIVE OPERATORS ${ }^{1,2}$
}

\author{
BY GIAN-CARLO ROTA
}

Communicated by I. M. Singer, August 12, 1961

The classical theory of Frobenius-Perron concerning the distribution of eigenvalues of a matrix with non-negative elements has been variously extended to positive operators, that is, linear operators on function spaces transforming non-negative functions into non-negative functions. Since the classical work of Jentzsch (see bibliography), there have been two kinds of extensions: (a) it has been established under various conditions that a positive operator shall have a positive eigenvector with positive eigenfunction (see e.g. Birkhoff [1], Karlin [4], Samelson [7], Schaefer [8]), and (b) attempts have been made to extend the Frobenius theorem stating that, for a non-negative matrix with spectral radius one, the eigenvalues on the unit circle are roots of unity. This result has been extended to positive operators under strong additional assumptions, all of them guaranteeing that the intersection of the spectrum with the unit circle shall consist of isolated points only. Results of this kind are summarized in Karlin. It is our present purpose to establish the second result under fairly general conditions. Our result is the following:

THEOREM. Let $P$ be a linear operator of norm at most one (a "contraction") in $L_{1}(S, \Sigma, \mu)$, where $(S, \Sigma, \mu)$ is a measure space of finite measure. Suppose that $P$ is bounded, with norm at most one, in $L_{\infty}(S, \Sigma, \mu)$. Then:

If $\alpha$ is an eigenvalue of $P$, and $P f=\alpha f$ for some nonzero integrable $f$, then $\alpha^{2}$ is also an eigenvalue of $P$.

If $f$ is written in the form $f=|f| g$, where the function $g$ is of absolute value one at every point, then the eigenfunction belonging to $\alpha^{2}$ is $|f| g^{2}$.

Operators of the type described in this theorem, the so-called dissipative operators, are commonly encountered in various circumstances, for instance in the study of semigroups generated by secondorder differential operators. The property of contractivity in $L_{1}$ is also crucially assumed in the pointwise ergodic theorems.

The proof of this theorem can be based upon elementary functional-analytic techniques, and will be given in full here. We begin by making the following two simplifications: (a) It can be assumed

\footnotetext{
${ }^{1}$ Work done by contract with the Office of Naval Research.

${ }^{2}$ I wish to thank Professor Jack Feldman of Berkeley for several enlightening conversations on this subject.
} 
that $\mu(S)=1$; and (b) by going over to the Stone-Čech compactification of $L_{\infty}(S, \Sigma, \mu)$, we can assume that all the bounded functions treated are continuous on a compact Hausdorff space. This second simplification is not strictly necessary, but it is convenient to talk about values of a function at a point. The proof results from the following seven steps.

(1) For a continuous non-negative function $f$, suppose that $P f=f$, then $P\left(f^{2}\right)=f^{2}$.

Indeed, for a fixed point $s$ in the compact Hausdorff space $S$, the linear functional $f \rightarrow P f(s)$ is positive and continuous. Hence, by the Riesz representation theorem, there exists a non-negative measure $m(s, d t)$ such that $P f(s)=\int f(t) m(s, d t)$ (integrations will always be carried out over the whole space). By Schwarz's inequality we have $\left(\int f(t) m(s, d t)\right)^{2} \leqq \int 1 m(s, d t) \int f(t)^{2} m(s, d t)$, where 1 is the function identically equal to one. Letting $P 1=h$, where $h$ is a non-negative function taking values between zero and one, the last inequality can be rewritten as $(P f(s))^{2} \leqq h(s)\left(P f^{2}\right)(s)$. But $P f=f$, hence this gives $f^{2} \leqq P\left(f^{2}\right)$. In view of the assumption that $P$ decreases the integrals of positive functions, this can only be true if $P\left(f^{2}\right)=f^{2}$.

(2) If $|P f|=|f|$, then $P|f|=|f|$, where $|f|$ is the function everywhere equal to $|f(s)|$. This is shown in the same way as (1), using the inequality $\left|\int f(t) m(s, d t)\right| \leqq \int|f(s)| m(s, d t)$.

(3) From (1) and (2) it follows easily that, if $P f=f$ and $P g=g$ for any two functions $f$ and $g$, then $P(f g)=f g$. Therefore, the fixpoints of $P$ form a subalgebra which, in view of the assumption, will be isomorphic to one of the form $L_{\infty}\left(E, \Sigma_{0}, \mu\right)$ on the original measure space, where $\Sigma_{0}$ is a $\sigma$-subfield of $\Sigma$, and $E$ a subset of $S$. In particular, the characteristic function (also known as the indicator) $\chi_{E}$ of the subset $E$ will belong to the subalgebra, that is $P \chi_{E}=\chi_{E}$.

(4) Now let $F$ be the complement of $E$ in the set $S$, and let $P \chi_{F}=q$. Then the function $q$ lives on $F$; for otherwise, $P 1=P\left(\chi_{E}+\chi_{F}\right)$ $>1$ at some point of $E$, contradicting the assumption that $P$ is a contraction in $L_{\infty}$. Hence the subspace $L_{1}(E, \Sigma, \mu)$, (where we denote by $\Sigma$ the $\sigma$-field obtained by intersecting the sets of $\Sigma$ with the set $E$ ) is invariant under $P$. Now, if $P f=\alpha f$ for some $\alpha$ of absolute value one, then, by (2), $P|f|=|f|$, hence $f$ lives on $E$. We conclude that it suffices to prove the theorem under the additional assumption that $P 1=1$, an assumption which we shall make from now on.

(5) If $P f=f$ and $g$ is arbitrary, then $P(f g)=f P g$. It suffices to prove this when $f \geqq 0$. Using the device of $(1)$, we have $\int f(t) m(s, d t)$ $=f(s)$ for each $s$. By Schwarz's inequality $\left(\int f(t) m(s, d t)\right)^{2}$ 
$\leqq \int f(t)^{2} m(s, d t)$. But by (1), equality holds in Schwarz's inequality. Hence $f(t)$ is, for fixed $s$, constant almost everywhere relative to the measure $m(s, d t)$. The assertion now becomes obvious when rewritten in terms of integrals relative to the measure $m(s, d t)$.

(6) Now let $P f=\alpha f$ for some $\alpha$ such that $|\alpha|=1$. Write $f=|f| g$, where $|g(s)|=1$ for all $s$ in its support, and let $G$ be the support of $f$. By (5), to complete the proof of the theorem, it suffices to prove that $P\left(g^{2}\right)=\alpha^{2} g^{2}$. Since the characteristic function of $G$ is invariant under $P$, we can restrict ourselves to functions living on $G$. Hence we shall assume that $|g(s)|=1$ for all $s$.

(7) The proof is now completed as follows. By Schwarz's inequality, $\left|\int g(t) m(s, d t)\right|^{2} \leqq \int|g(t)|{ }^{2} m(s, d t)=1$. But, since $|P g|=|g|$. equality holds in the inequality, and this can only happen if $g(t)$ is constant almost everywhere relative to the measure $m(s, d t)$. In view of the identity $\int g(t) m(s, d t)=\alpha g(s)$, it follows that, for fixed $s$, $g(t)=\alpha g(s)$ for a set of points $t$ of $m(s, d t)$-measure one. But if this is the case, then also $g(t)^{2}=\alpha^{2} g(s)^{2}$ for a set of points of $m(s, d t)$ measure one. Integrating, we obtain $\int g(t)^{2} m(s, d t)=\alpha^{2} g(s)$, that is, changing notation, $P\left(g^{2}\right)=\alpha^{2} g^{2}$, q.e.d.

We conjecture that the assumptions of the above theorem are essentially sharp, that is, any significant class of operators for which the assertion holds is equivalent, after a change of measure, to the one considered above. For example, it is easy to see that those quasicompact operators for which the assertion is true are equivalent, after a change of measure, to contractions.

\section{BIBLIOGRAPHY}

1. Birkhoff, Extensions of Jentzsch's theorem, Trans. Amer. Math. Soc. vol. 85 (1957) pp. 219-227.

2. Frobenius, Ueber Matrizen aus positiven Elementen, S.-B. Preuss. Akad. Wiss. Berlin (1908) pp. 471-476, (1909) pp. 514-518, (1912) pp. 456-477.

3. Jentzsch, Ueber Integralgleichungen mit positiven Kern, Crelle's Journal vol. 141 (1912) pp. 235-244.

4. Karlin, Positive operators, J. Math. Mech. vol. 8 (1959) pp. 907-938.

5. Perron, Zur Theorie der Matrizen, Math. Ann. vol. 64 (1907) pp. 248-263.

6. Putnam, On bounded matrices with non-negative elements, Canad. J. Math. vol. 10 (1958) pp. 587-591. 59.

7. Samelson, On the Perron-Frobenius theorem, Michigan Math. J. (1957) pp. 57-

8. H. Schaefer, Some spectral properties of positive linear operators, Pacific J. Math. vol. 10 (1960) pp. 1009-1019.

9. J. T. Schwartz, Eigenvalues of postive compact operators, to appear in Communications PAM.

MassachusetTs Institute of Technology 